$\underline{\text { Review Article }}$

\title{
RECENT NANOCOCHLEATE DRUG DELIVERY SYSTEM FOR CANCER TREATMENT: A REVIEW
}

\author{
SUJIT NAYEK*, ABHIRAMI VENKATACHALAM, SANGEETA CHOUDHURY
}

Department of Pharmaceutics, Acharya and BM Reddy College of Pharmacy, Soldevanahalli, Hesaraghatta Road, Bangalore 560107, Karnataka, India

Email: sujitnayek29@gmail.com

Received: 15 Aug 2019, Revised and Accepted: 12 Oct 2019

\begin{abstract}
Nanocochleates are at the forefront of the fast-growing nanotechnology sector in the delivery of drugs for cancer. This nanotechnology is the use of the cationic and anionic encapsulated drug that has poor oral bioavailability. Nanocochleate is a lipid-based drug delivery in the liposomal vesicles that is converted by calcium divalent cation into nanocochleate. Nanocochleates technology use encapsulations of the anticancer agent, which have low solubility, oral bioavailability and low permeability. This paper shows and provides an overview of the benefits of nanocochleates, drug delivery mechanism, choice of prevalent components (Phospholipids and Cations), various ways of producing nanocochleates and nanocochleate stability. Nanocochleates have far fewer constraints than other traditional carriers. To characterize nanocochleates, the suitable analytical methods are required. Therefore, in the therapy of cancer, nanocochleate becomes commonly applied and more prospective drug delivery system.
\end{abstract}

Keywords: Nanocochleates, Cancer therapy, Cochleate, Phosphatidylserine, Cationic drug

(c) 2019 The Authors. Published by Innovare Academic Sciences Pvt Ltd. This is an open access article under the CC BY license (http://creativecommons.org/licenses/by/4.0/) DOI: http://dx.doi.org/10.22159/ijcpr.2019v11i6.36359

\section{INTRODUCTION}

Cancer continues to be a serious threat with higher mortality risk. Cancer is a disease classified by an uncontrolled cell proliferation that can invade and spread to distant parts of the body through the lymphatic system [1]. The goal of the ideal cancer chemotherapy is to deliver the right amount of drug to the site of action with the desired controlled rate and long duration of time. The death rate from cancer worldwide is estimated at 9.6 million fatalities in 2018, according to the World Health Organization (WHO) [2]. The American Cancer Society estimates approximately 17,35,350 new diagnosed cancer cases and 6,09,640 deaths by 2018 in the United States [3]. In India, the cancer death rate in 2018 is estimated at around 7,84,821 fatalities [4]. The above statistics indicate that cancer disease needs to be controlled.

Nanomedicine emerged from nanotechnology, as predicted by Richard P. Feynman, it is rather a nascent science field from 1959 [5]. Nanoparticles have proven to be one of the logical and promoting carriers for the controlled and targeted delivery of medicine. Nanomedicine plays a major role in overcoming this challenge as formulations based on nanotechnology improve bioavailability, pharmacokinetic characteristics and drug targeting in different disorders. Nanotechnology increases the acceptability and performance of dosage forms by increasing their safety, effectiveness, patient faithfulness and eventually lowering the price of health care [6-8].

Due to their low drug loading capacity, poor mechanical stability, high production costs, phospholipids sometimes undergo oxidation and hydrolysis as a short half-life reaction; liposomes are not able to improve drug oral absorption. So, to solve the above problem, there is a necessitate to develop such a drug delivery system. Cochleates are elongated rolled microstructures composed of a sequence of lipid bilayers created by condensation of negative charged small unilamellar liposomes. Papahadjopoulos and Wilschut first reported these structures at 1979. Nanocochleates carrier is based on the encapsulation of drugs in a multilayered lipidic matrix for safe and effective delivery of the drugs in cancer cells [9-13].

\section{Basics of nanocochleates}

Nanocochleates are microstructures similar to cigars that consist of a sequence of lipid bilayers. These lipid-based carriers are stable precipitates of phospholipid cation consisting of naturally occurring materials phosphatidylserine and calcium ion in general. In the formulation of nanocochleate continuous lipid bilayers sheet rolled up in a way that developed spiral structure without inner aqueous phase between them cochleares differing from liposomes in having cylindrical shaped and water-free interior structure [9-11].

Nanocochleates are novel lipid-based drug delivery carriers compared to traditional drug carriers for many reasons such as (i) they are more stable than liposome due to less oxidation of lipid, (ii) they are non-immunogenic, non-inflammatory and non-toxic, (iii) nanocochleates maintain their structure even after lyophilization, whereas liposomes structures are destroyed after lyophilization, (iv) improvement of oral bioavailability of hydrophilic and hydrophobic drugs, (v) they exhibits the adequate encapsulation of hydrophobic drugs in to lipid bilayers structure [14-16].

Although these benefits, nanocochleate suffers from certain constraints, such as (i) required particular storage condition, (ii) aggregation may happen at times during storage, (iii) high production costs $[15,16]$.

\section{Mechanism of drug delivery}

\section{Absorption from intestine}

Nanocochleate absorption from the intestine occurs after oral administration. Cross the digestive epithelium, nanocochleates bring their active agents to the blood vessel.

In the case of other routes except intravenous, they cross and enter into circulation through the epithelium cell. They are supplied to the targeted cell after they reach into circulation [10].

\section{Delivery to targeted cells}

There has been an extensive study of calcium interaction with negatively charged lipids. Many natural fusion events require calcium interaction with negatively charged phospholipids (usually phosphatidylglycerol or phosphatidylserine). In many natural membrane-fusion procedures, calcium-induced disturbances of membranes containing negative charged lipids and subsequent membrane-fusion occurrences are significant mechanisms. Therefore, cochleate can be considered as an intermediate membrane-fusion $[10,11,14,17]$.

\section{Delivery by cell membrane fusion}

First, nanocochleate comes near to the cell membrane, triggering cell membrane disruption and reordering, leading to a fusion event 
between the exterior layer of the nanocochleate and the membrane of the cell. This fusion results in a tiny quantity of the encochleated material being delivered into the target cell's cytoplasm. This carrier can slowly fuse or break free from the cell and be accessible with this or another cell for another fusion case [14, 17].

\section{Structure and components of nanocochleate drug delivery system}

Nanocochleate is a cigar-like structure consisting of a constant bilayer lipid. Cigar-like structure created by tiny unilamellar anionic liposome condensation. A rolled cochleate traverse segment is provided in fig. 1. The main components used in nanocochleate preparing are lipids and cations as shown in table 1.

\section{Methods of preparation}

Nanocochleates are prepared using various techniques such as Hydrogel Method, Trapping method, Liposomes before cochleate dialysis technique, direct calcium dialysis technique and Binary aqueous-aqueous emulsion system.

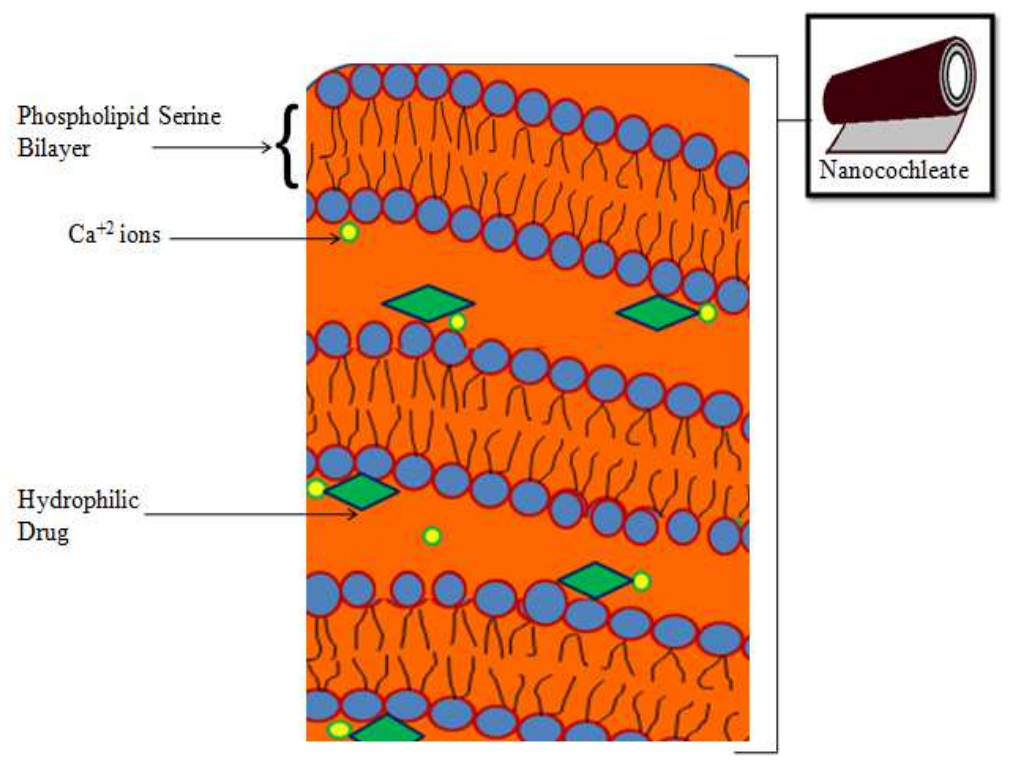

Fig. 1: Traverse section of rolled cochleate

Table 1: Major ingredients to make nanocochleates

$\left.\begin{array}{lll}\hline \text { Ingredients } & \text { Ref. } \\ \hline \text { Lipids } & \text { Phosphatidic Acid, Phosphatidyl Ethanolamine, Phosphatidyl Serine, Phosphatidyl Glycerol, Di-Myristoyl Phosphatidyl Serine, } & {[10-} \\ & \begin{array}{l}\text { Di-Oleoyl Phosphatidyl Serine, Phosphatidyl Inositol, Di-Stearoyl Phosphatidyl Serine, Phosphatidyl Choline, Di-Phosphatidyl } \\ \text { Glycerol, Di-Oleoyl Phosphatidic Acid, Di Palmitoyl Phosphatidyl Gycerol. }\end{array} & 12, \\ \text { Cations } & \mathrm{Mg}^{+2} \text { or Ca } & \text { 18 } \mathrm{Zn}^{+2} \text { or Ba }\end{array}\right]$

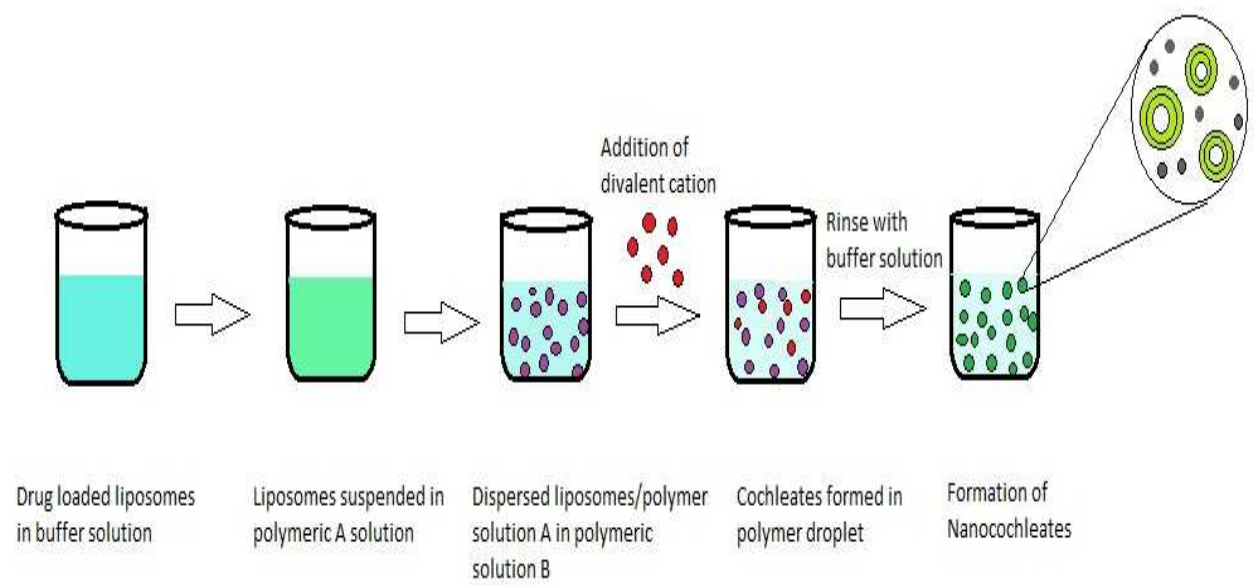

Fig. 2: Hydrogel method

\section{Hydrogel method}

Standard techniques such as sonication or micro fluidization are used to prepare a suspension of tiny unilamellar liposomes. The suspension of the liposome is combined with polymer A like dextran, phosphatidylserine or polyethylene glycol. By injection, the liposomes/polymeric A solution is added to a new polymeric B solution, such as polyvinyl alcohol, polyvinyl pyrolidone, polyvinyl methyl ether and ficoll, where polymer A is immiscible, resulting in an aqueous two-phase polymer scheme. By using a syringe pump at a regulated pace of $1 \mathrm{ml} / \mathrm{min}$ to $50 \mathrm{ml} / \mathrm{min}$, this can be accomplished mechanically. 
The above two-phase system is supplemented with a solution of cation salt. The divalent cation spreads into polymeric $\mathrm{B}$ and then into the particles consisting of liposomes/polymeric A enabling the development of tiny sized cochleates. Cochleates are separated and washed constantly with a buffer that contains a molecule that is positively charged, i.e. divalent cation. Adding cations to the wash buffer guarantees that the cochleate structures stay as precipitated during the entire washing process $[19,20]$. The above method is shown in fig. 2.

\section{Trapping method}

Trapping method includes the development of liposomes of phosphatidyl-serine followed by the addition of calcium chloride. Either adding the water phases to a phospholipid film or adding water to phospholipid powder can generate liposomes. In continuous vortexing, calcium chloride solution $(3 \mathrm{mmol})$ is added to $1 \mathrm{ml}$ of liposomal small unilamellar vesicle dispersion to form cochleates. A precipitate that is cooled at 2 to $8{ }^{\circ} \mathrm{C}$ is created $[21,22]$. The above method is shown in fig. 3.

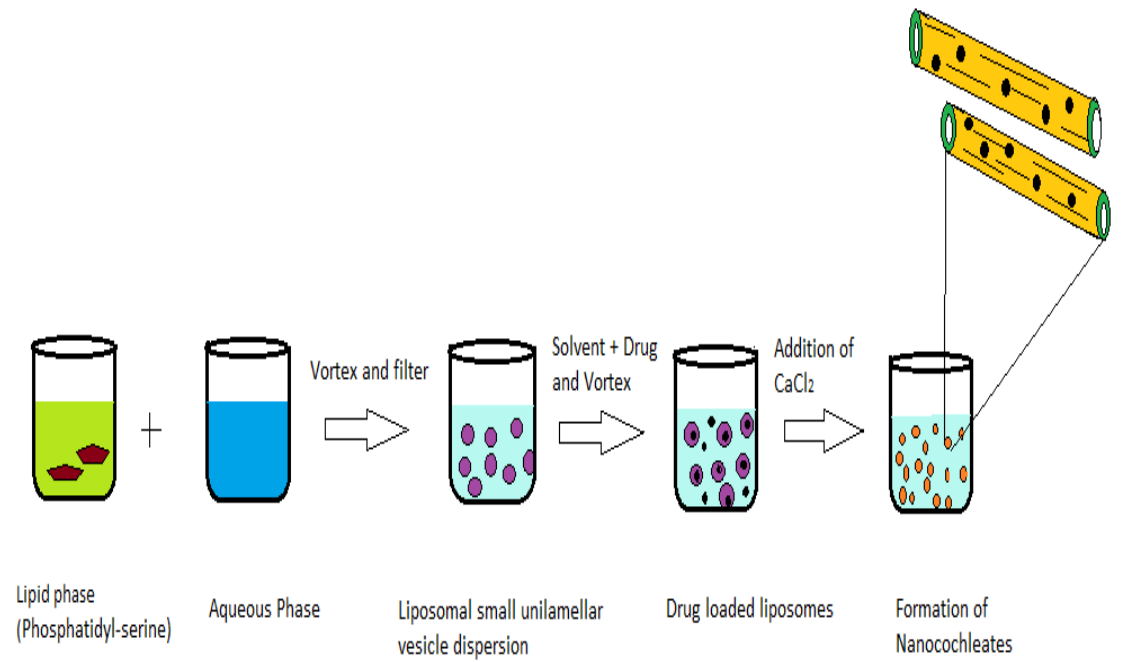

Fig. 3: Trapping method

Poudel et al. developed docetaxel loaded nanocochleates for controlled release of drugs, improved bioavailability, targeted delivery, and increased anticancer potential. Trapping method was used to prepare nanocochleates. In docetaxel loaded liposomal formulation under vortex, calcium chloride $(20 \mu \mathrm{l})$ was added dropwise. The liposomal phase turned turbid instantly due to the creation of nanocochleates. Nanocochleates loaded docetaxel formulation were stored at 2 to $8^{\circ} \mathrm{C}$ [23].

\section{Liposomes before cochleates dialysis technique}

An aqueous suspension is prepared containing a lipid detergent combination. The suspension of the lipid-detergent is blended with polymer A like phosphatidylserine, polyethylene glycol or dextran. The lipid-detergent/polymer A suspension is introduced to a solution consisting of polymer B (e. g. polyvinyl alcohol, polyvinyl pyrolidone, polyvinyl methyl ether and ficoll) in which both polymers are immiscible, producing a polymer system of two phases. A cationic moiety solution is introduced to the polymer structure in two phases. Now wash the polymer system in two phases to remove the polymer and the cochleate created [20,24].

\section{Direct calcium dialysis technique}

The mixture of cholesterol and phosphatidylserine (1:9 weight ratios) in the non-ionic detergent and buffer is combined with a pre-selected polynucleotide concentration. The above solution is vortexed for 5-10 min. The resulting colorless solution is dialyzed against three buffer modifications at room temperature. The concentrations of $\mathrm{Ca}+2$ (6 $\mathrm{mmol}$ ) and buffer are consistent with cochleate formation. The resulting white precipitates of calcium-phospholipid are called direct calcium cochleate. Very large sized cochleates are formed [22, 24].

\section{Binary aqueous-aqueous emulsion system}

Small unilamellar vesicle liposomes are produced either by film or high $\mathrm{pH}$ method in this technique. Then a polymer like dextran mixes the liposomes. A second non-miscible polymer (e. g. Polyethylene glycol) is injected into the dextran/liposome phase. Calcium is then added and spread slowly from one phase to the next forming cochleates, after which the gel is washed away. Cochleates are formed by this technique with particle size less than $1000 \mathrm{~nm}[20,25]$.

\section{Stabilization of nanocochleates}

Nanocochleates are stable formulations of lipid-based delivery with very different structures and properties from liposomes. Lyophilization is a promising way of increasing the nanocochleate's chemical and physical stability over time. It is expected that the solidstate of lyophilizates will have better physical and chemical stability than aqueous lipidic dispersions. To protect the nanoparticulate suspension, the most common cryoprotective agents such as sorbitol, mannose, trehalose, and glucose are added [26]. Nanocochleates can be lyophilized to a powder form and stored at a temperature of $40^{\circ} \mathrm{C}$. Lyophilized cochleate may be reconstituted with liquid before in vitro use or in vivo administration. Lyophilization does not adversely affect the morphology or functions of cochleate [27].

\section{Characterization techniques of nanocochleates}

\section{Particle size determination}

Laser diffraction technique is used to determine the mean particle size of the cochleate dispersion and liposomal dispersion. Analysis is performed at a detection angle of $90^{\circ}$ at $30 \pm 2{ }^{\circ} \mathrm{C}$ [28].

\section{Entrapment efficiency (\%) determination}

Cochleates $(100 \mu \mathrm{l})$ are aliquoted into centrifuged tubes. During vortexing, $1 \mathrm{ml}$ of ethanol and $60 \mu \mathrm{l} \mathrm{pH} \mathrm{9.5} \mathrm{EDTA} \mathrm{solutions} \mathrm{are} \mathrm{added}$ [29]. The absorbance of the solution is measured using a suitable analytical technique and entrapment efficiency is calculated using the following equation-

Entrapment efficiency $(\%)=\frac{\text { Amount of drug present in cochleate }}{\text { Total amount of drug present }}: 100$

\section{Surface morphology study}

Nanocochleate surface morphology is performed using transmission electron microscopy. A drop of the diluted sample is placed on a carbon-coated copper grid to form a thin liquid film in order to 
prepare the sample for this study. The sample is examined and photographed at an accelerating voltage of $80 \mathrm{kV}$ with a transmission electron microscope after excess solution is removed [30].

\section{Fourier transform infrared spectroscopy study}

Fourier transform infrared spectroscopy study determines the functional groups present as well as the purity of the compound. Samples are prepared by mixing with $\mathrm{KBr}$. Then samples are placed in the holder. The spectra are scanned at ambient temperature over the particular ranges of wave numbers [28].

\section{Differential scanning calorimetry study}

Differential scanning calorimetry study determines the lipid status. The samples are packed hermetically in perforated aluminum pans and heated over the temperature range of -10 to $180{ }^{\circ} \mathrm{C}$ at a steady pace of $10{ }^{\circ} \mathrm{C} / \mathrm{min}$. The system is purged at a speed of $100 \mathrm{ml} / \mathrm{min}$ with nitrogen gas to keep the atmosphere inert [28, 31].

\section{Cochleates-cell interaction study}

In this study, approximately $2 \%$ fluorescent lipid is used to create fluorescent liposomes to examine the interaction of cochleate with cell membranes. When cochleate interacts with fluorescent membranes, cell surfaces under fluorescent microscopes become fluorescent.

\section{Surface charge determination}

The nature and intensity of nanocochleate's surface charge determines its interaction with the biological environment and its electrostatic interaction with bioactive compounds. By measuring the particle velocity in an electrical field, the surface charge can be determined. Laser light scattering techniques like Velocimetry or Laser Doppler Anemometry are used to determine the velocities of nanocochleates [32].

\section{In vitro release study}

\section{Diffusion cell method}

In diffusion cell method, double chamber diffusion cells on a shake stand is usually used. Between the two chambers there is a millipore, low protein binding membrane. The receptor chamber is filled with phosphate buffer. The donor chamber is filled with formulation and standard analytical methods are used to test the receptor compartment for the released drug at different time intervals.

\section{Modified ultra-filtration method}

This method is also used to determine the released drug concentration of nanocochleates. Here nanocochleates are introduced directly to a buffer-containing stirred ultra-filtration cell. Aliquots of the medium are filtered at different time intervals through the membrane. Then, the drug concentration is determined using any suitable analytical methods [15].

\section{Stability study}

To check stability, cochleates dispersions can be maintained at a temperature of 2 to $8{ }^{\circ} \mathrm{C}$ and relative humidity of $25 \pm 2{ }^{\circ} \mathrm{C} / 60 \%$ for a period of 3 mo. The stability of the formulation is determined in terms of change in entrapment efficiency (\%) and particle size [28].

\section{CONCLUSION}

Nanocochleates are a drug delivery carrier based on lipids that show oral potential in the delivery of anticancer drugs. Nanocochleate drug delivery systems can, therefore, be used as an alternative to delivering the biological or therapeutic agents to the cancer cells in the future. Nanocochleate application can improve the real effectiveness of chemotherapeutic agents in cancer treatment. Definitely nano cocreates can push the pharmaceutical world into the fresh age of delivering extremely difficult drugs to cancer drugs.

\section{AUTHORS CONTRIBUTIONS}

All the author have contributed equally.

\section{CONFLICTS OF INTERESTS}

The authors declare no conflicts of interest

\section{REFERENCES}

1. Mei L, Zhang Z, Zhao L, Huang L, Yang XL, Tang J, et al. Pharmaceutical nanotechnology for oral delivery of anticancer drugs. Adv Drug Delivery Rev 2012;65:880-90.

2. Cancer statistics report worldwide. World Health Organization Web. Available from: https://www.who.int/news-room/factsheets/detail/cancer. [Last accessed on 24 Aug 2019]

3. Cancer Facts Figures. American cancer society. Available from: https://www.cancer.org/content/dam/cancer$\mathrm{org} /$ research/cancer-facts-andstatistics/annual-cancer-factsand-figures/2018/cancer-factsand-figures-2018.pdf. [Last accessed 24 Aug 2019]

4. Cancer statistics report in India. Cancer India. Available from: http://cancerindia.org.in/cancer-statistics/. [Last accessed on 15 Sep 2019]

5. Feynman RP. There's plenty of room at the bottom. Eng Sci 1960;23:22-36.

6. Freitas RA. The future of nanofabrication and molecular-scale devices in nanomedicine. Stud Health Technol Inform 2002;80:45-59.

7. Kim B, Rutka JT, Chan WC. Nanomedicine. N Engl Med 2010;363:2434-43.

8. Feng SS, Zhao L, Tang J. Nanomedicine for oral chemotherapy. Nanomed 2011;6:407-10.

9. Pawar AY, Jadhav KR, Sonkamble NB, Kale MR. Nanocochleate: a novel drug delivery system. Asian J Pharm 2016;10:S234-42.

10. Bothiraja C, Rajput N, Poudel I, Rajalakshmi S, Panda B, Pawar A. Development of novel biofunctionalized chitosan decorated nanocochleates as a cancer-targeted drug delivery platform. Artif Cell Nanomed B 2018;25:1-15.

11. Pawar A, Bothiraja C, Shaikh K, Mali A. An Insight into cochleates, a potential drug delivery system. RSC Adv 2015;5:81180-202.

12. Yeale SE, Chadhari P. A review on nanocochleate-a novel lipidbased drug delivery system. J Biomed Pharm Res 2013;2:53955.

13. Wicki A, Witzigmann D, Balasubramanian V, Huwyler J. Nanomedicine in cancer therapy: challenges, opportunities, and clinical applications. J Controlled Release 2015;200:138-57.

14. Harke B, Kulkarni A, Allorkar N. Nanocochleate: a new cornucopia in oral drug delivery. Int J Innov Pharm Sci 2013;2:1-9.

15. Bhosale R, Ghodake PP, Mane AN, Ghadge AA. Nanocochleates: a novel carrier for drug transfer. J Sci Innov Res 2013;2:964-9.

16. Vijeta $P$, Vivek M, Panwar AS, Darwhekar GN, Jain DK. Nanocochleate: as drug delivery vehicle. Int J Pharm Biol Sci 2011;1:31-8.

17. Anantharaman NV, Udhumansha U, Rathnam G. Optimization of cytarabine loaded nanocochleates for targeting leukemia by response surface methodology. Int J Res Pharmacol Pharmacother 2018;7:205-21.

18. Elsayed M, Abdallah OY, Naggar VF, Khalafallah NM. Lipid vesicles for skin delivery of drugs: reviewing three decades of research. Int J Pharm 2007;332:1-16.

19. Zarif L, Jin T, Segarra I, Mannino RJ. Hydrogel isolated cochleate formulations, the process of preparation and their use for the delivery of biologically relevant molecules. U. S. Patent 6592894B1; 2003.

20. Mannino RJ, Chanki M, Feketeova E, Scolpino AJ, Wang Z, Kheriri MT, et al. Targeting immune response induction with cochleate and liposome-based vaccines. Adv Drug Delivery Rev 1998;32:273-87.

21. Sonwane SA, Chavan MJ, Hase DP, Chumbhale DS, Ambare AS, Bodakhe T. Preparation, characterization and in vitro anticancer testing of quercetin-loaded nanocochleates. Pharm Res 2017;1-7.

22. Jin T, Zarif L, Mannino R. Nanocochleate formulations, the process of preparation and method of delivery of pharmaceutical agents. U. S. Patent 6153217A; 2000. 
23. Poudel I, Ahiwale R, Pawar A, Mahadik K, Bothiraja C. Development of novel biotinylated chitosan-decorated docetaxel-loaded nanocochleates for breast cancer targeting. Artif Cells Nanomed Biotechnol 2018;46:22940.

24. Zarif L. Elongated supramolecular assemblies in drug delivery. J Controlled Release 2002;81:7-23

25. Sankar VR, Reddy YD. Nanocochleate-a new approach in lipid drug delivery. Int J Pharm Pharm Sci 2010;2:220-3

26. Souto EB. SLN and NLC for topical delivery of antifungals [dissertation]. Free University of Berlin; 2005.

27. Delmarre D, Lu R, Tatton N, Krause ES, Gould Fogerite S, Mannino R. Formulation of hydrophobic drugs into cochleate delivery vehicles: a simplified protocol and formulation kit. Drug Delivery Technol 2004;4:64-9.
28. Landge A, Pawar A, Shaikh K. Investigation of cochleates as carriers for topical drug delivery. Int J Pharm Pharm Sci 2013;5:314-20.

29. Leila Zarif. Drug delivery by lipid cochleates. In: Duzgunes N. editor. Methods in Enzymology, Liposomes. Part E. United Kingdom: Academic Press; 2005. p. 314-29.

30. Pawar AP, Vinugala D, Bothiraja C. Nanocochleates derived from nanoliposomes for paclitaxel oral use: preparation, characterization, in vitro anticancer testing, bioavailability and biodistribution study in rats. Biomed Pharmacother 2014;1-9. Doi:10.1016/j.biopha.2014.11.014.

31. Westesen K, Siekmann B, Koch MHJ. Investigations on the physical state of lipid nanoparticles by synchrotron radiation $\mathrm{x}$ ray diffraction. Int J Pharm 1993;93:189-99.

32. Gol D, Shah V. Nanocochleates: a novel approach for drug delivery. World J Pharm Res 2014;3:1920-44. 